\title{
Ultrasonic Testing of Engineering Plastics Using Aluminum Powder Coupling
}

\author{
Kazuya Mori $^{\mathrm{a},{ }^{*}}$, Teruyuki Mori ${ }^{\mathrm{b}}, \mathrm{Kenji} \mathrm{Hashimura}^{\mathrm{a}}$, Kazuhisa Era $^{\mathrm{a}}$, Kei Yamashita $^{\mathrm{c}}$ \\ ${ }^{a}$ Kumamoto University, 2-39-1 Kurokami, Chuo-ku, Kumamoto, 860-8555, Japan \\ ${ }^{b}$ Ryoyu System Engineering Co., Ltd., 1106-4 Shimizu, Uozumi, Akashi, 674-8686, Japan \\ ${ }^{\mathrm{c}}$ Bridgestone Corporation, 1-1, Kyobashi 3-chome, Chuo-ku, Tokyo 104-8340, Japan
}

*Corresponding Author: kmori@ @ech.kumamoto-u.ac.jp

\begin{abstract}
A new immersion testing method has been developed where the water of the conventional method has been replaced with powder, specifically in this study aluminum powder. In this new method, objects are ultrasonically tested while immersed in pressurized powder. In this paper engineering plastics were tested using this method. The acoustic impedance of the aluminum powder and the test objects match each other greatly reducing the elastic wave reflections.
\end{abstract}

Keywords: ultrasonic testing, powder immersion testing, engineering plastic, aluminum powder.

\section{Introduction}

Immersion testing is one type of ultrasonic testing $\operatorname{method}^{(1)}$. The sound waves from a transducer are coupled to a test object with water. This can be done by immersing the transducer and the test object in a tank of water. However, the acoustic impedance of test objects differs widely from each other and creates echoes. These echoes inhibit the detection of defects. It is difficult to detect defects just below the surface of test objects as well as thin objects.

A method of matching the acoustic impedance of the coupling and the test object has been developed. The water coupling of the conventional method has been replaced with powder because powder has higher acoustic impedance which can match that of objects. We call this new method "Powder Immersion Testing." This testing method has been applied to engineering plastics and the effectiveness of the method has been verified.

\section{Powder immersion testing}

According to the basic elastic wave theory which is shown in Fig. 1. ultrasonic waves enter an interface between two solid mediums. At the interface the ultrasonic incident waves are partially reflected from the interface and partially transmitted through the interface ${ }^{(2)}$. The coefficient of reflection $R$ which is the ratio of the acoustic pressure of the reflected wave, $P_{r}$ to that of the incident wave, $P_{i}$, and the coefficient of transmission $T$ which is the ratio of the pressure of the transmitted wave, $P_{t}$ to that of the incident wave, $P_{i}$, are given by

$$
R=\frac{P_{r}}{P_{i}}=\frac{Z_{2}-Z_{1}}{Z_{2}+Z_{1}}
$$

$$
T=\frac{P_{t}}{P_{i}}=\frac{2 Z_{2}}{Z_{2}+Z_{1}}
$$

where $Z_{1}$ is the acoustic impedance of the region in which the wave is approaching the interface, $Z_{2}$ is the acoustic impedance of the region beyond the interface.

When the region beyond the interface is a specimen assuming the acoustic impedance of the incident side region, $Z_{1}$ matches the acoustic impedance of the specimen, $Z_{2}$,

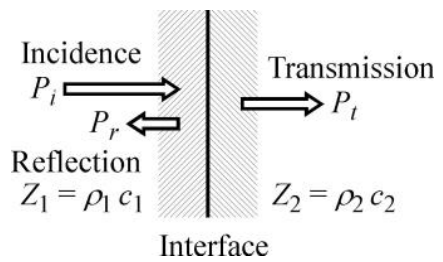

Fig. 1. Transmission and reflection of ultrasonic wave at an interface. 
the reflectance $R$ becomes zero. Namely, there is no reflected wave and we are able to test small or thin specimens as well as specimens with rough surfaces.

Based on the theorem mentioned above, the authors developed the "Powder Immersion Testing" as shown in Fig. 2. where the water of the conventional immersion testing method is replaced with powder. Even though the powder usually prevents the transmission of elastic waves, they can be transmitted through it under pressurized conditions. The advantages are as follows;

(a) Acoustic impedance of powders is generally high in comparison with those of liquids and is able to match the acoustic impedance of engineering materials.

(b) Acoustic impedance of powders can be changed by controlling the pressure applied to the powder. Therefore, it is easy to make the acoustic impedance of the powder match that of engineering materials.

(c) Pressurized powder and rough surfaces may be integrated acoustically and rough surfaces can be tested as if smooth.

(d) Ultrasonic testing is applied while specimens are dry. As a result materials which might be affected by water can also be tested.



Fig. 2. Powder immersion testing
The disadvantages of powder immersion testing are as follows;

(a) High pressure may be required so that ultrasonic waves penetrate powder which requires extra equipment.

(b) Tested objects may be broken by the applied pressure.

(c) It may be difficult to obtain high acoustic impedances that might be required in testing heavy metals because of the high pressures involved.

\section{Acoustic Impedance of Engineering Plastic and Aluminum Powder}

\subsection{Acoustic Impedance of Engineering Plastics}

Prior to using powder immersion testing, the acoustic impedance of the test objects and the powder are required. With this information we can then choose a powder which best matches that of the test material. Acoustic impedance of mediums, $Z$ is given by

$$
Z=\rho \times c
$$

where $\rho$ and $c$ are the density and the velocity of elastic wave through the medium.

The velocity of the longitudinal waves is given by

$$
c=\sqrt{\frac{E(1-v)}{\rho(1+v)(1-2 v)}}
$$

where $E$ and $v$ are the Young's modulus and the Poisson's ratio of the medium

From Eqs. (3) and (4), we obtain the acoustic impedance of longitudinal waves;

$$
Z=\sqrt{\frac{\rho E(1-v)}{(1+v)(1-2 v)}}
$$

The material properties, the velocities of longitudinal wave and the acoustic impedance obtained from Eq. (4) and Eq. (5) are given in Table 1. for typical engineering plastics.

\begin{tabular}{|c|c|c|c|c|c|c|}
\hline \multirow[t]{3}{*}{ Material } & \multirow[t]{3}{*}{ Symbol } & Density & $\begin{array}{l}\text { Young's } \\
\text { modulus }\end{array}$ & $\begin{array}{l}\text { Poisson's } \\
\text { ratio }\end{array}$ & $\begin{array}{l}\text { Velocity of } \\
\text { longitudinal } \\
\text { wave }\end{array}$ & $\begin{array}{l}\text { Acoustic } \\
\text { impedance }\end{array}$ \\
\hline & & $\rho$ & $E$ & $v$ & $c_{\mathrm{p}}$ & $Z$ \\
\hline & & $\left(\mathrm{kg} / \mathrm{m}^{3}\right)$ & $(\mathrm{GPa})$ & $(-)$ & $(\mathrm{m} / \mathrm{s})$ & (MPa s/m) \\
\hline polyacetal & POM & 1410 & 2.6 & 0.35 & 1720 & 2.4 \\
\hline polyamide & PA & 1130 & 2.5 & 0.41 & 2268 & 2.6 \\
\hline polycarbonate & $\mathrm{PC}$ & 1200 & 2.35 & 0.38 & 1915 & 2.3 \\
\hline modified polyphenylene ether & m-PPE & 1060 & 2.45 & 0.36 & 1971 & 2.1 \\
\hline polybutylene terephthalate & PBT & 1310 & 2.6 & 0.37 & 1874 & 2.5 \\
\hline
\end{tabular}
The material properties are typical values when it is around

Table 1. Acoustic Impedance of Engineering Plastics 
$300 \mathrm{~K}$. It can be seen from Table 1. that the acoustic impedance of typical engineering plastics ranges from 2.1 $\mathrm{MPa} \mathrm{s} / \mathrm{m}$ to $2.6 \mathrm{MPa} \mathrm{s} / \mathrm{m}$.

\subsection{Acoustic Impedance of Aluminum Powder}

The acoustic impedance of aluminum powder was measured experimentally using a powder immersion testing vessel as shown in Fig. 2. Two types of aluminum powder were used; one fine (A32) and one course (A21) as shown in Table 2. The acoustic impedance of each powder can be obtained from its density and the velocity of the elastic wave seen in Eq. (3). The velocity can be measured from the traveling time of the elastic wave in the vessel.

The density, $\rho$ can be obtained by

$$
\rho=\frac{m}{A h}
$$

where $m$ is the mass of powder, $A$ is the cross sectional area

Table 2. Mean grain size of aluminum powder

\begin{tabular}{|c|c|c|}
\hline & Product name & Mean grain size $(\mu \mathrm{m})$ \\
\hline Fine aluminum powder & A32 & 1 \\
\hline Coarse aluminum powder & A21 & 80 \\
\hline
\end{tabular}

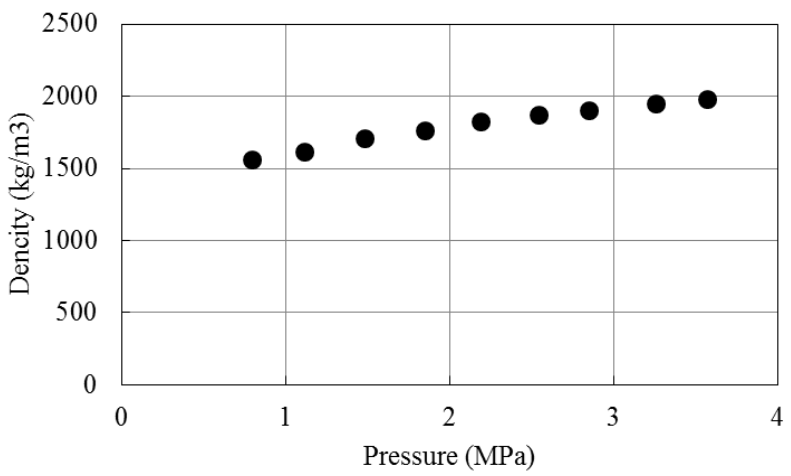

Fig. 3. Density of the aluminum fine powder A32

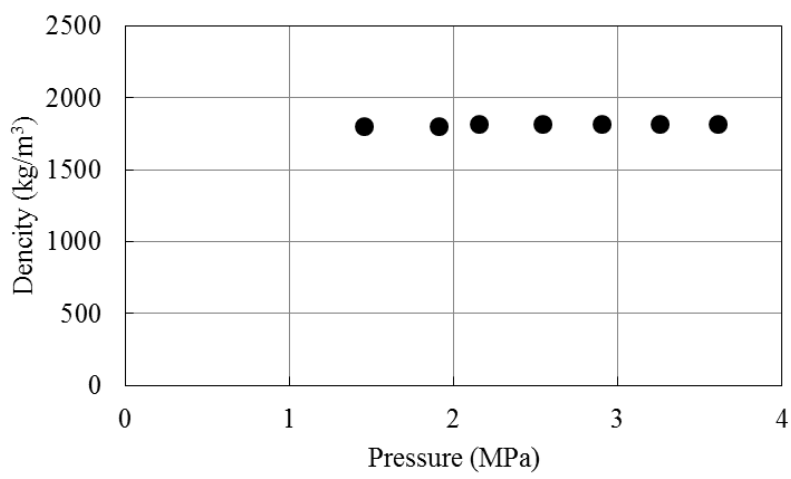

Fig. 4. Density of the aluminum coarse powder A21 of the pressure vessel, and $h$ is the height of the powder.

The sound velocity, $c$ can be obtained by

$$
c=\frac{2 h}{t}
$$

where $t$ is the reflection time and the travel path of the ultrasonic wave is $2 h$.

Substituting Eqs. (6) and (7) into Eq. (3), we obtain

$$
Z=\frac{2 m}{A t}
$$

Figures 3. and 4. show the densities of the aluminum powder, A32 and A21 versus the densities under pressure respectively. The density of A32 is increased in relation to the pressure while that of A21 is constant.

Figures 5. and 6. show the sound velocities of A32 and A21. The velocities are also showing the same tendency. The zero values in Fig. 5. represent no echo measurement.

The acoustic impedance, $Z$ can be obtained from Figs. 3. to 6. and Eq. (3). Figures 7. and 8. show the acoustic impedance of aluminum powder A32 and A21.

\section{Powder Immersion Testing of Engineering Plastics}

Polycarbonate specimens have been tested using powder immersion testing. The acoustic impedance of the

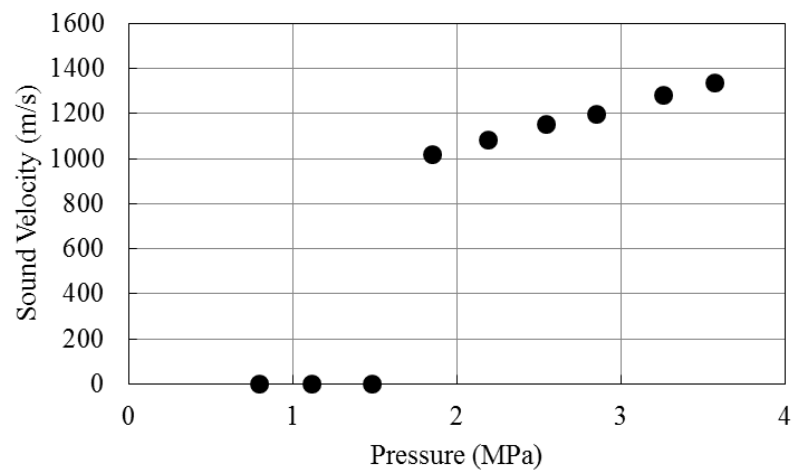

Fig. 5. Sound velocity of the aluminum fine powder A32

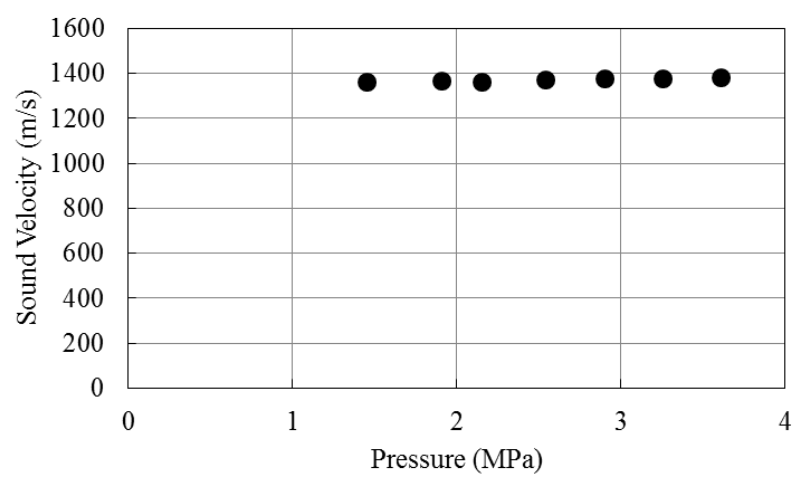

Fig. 6. Sound velocity of the aluminum coarse powder A21 


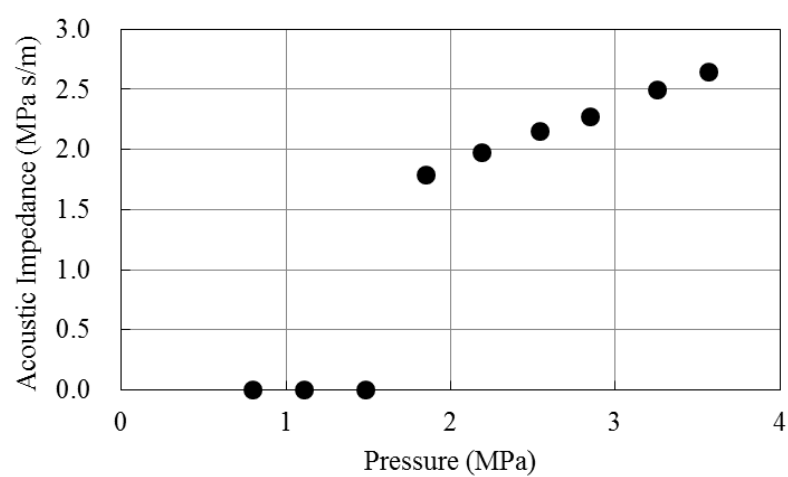

Fig. 7. Acoustic impedance of the fine aluminum powder A32

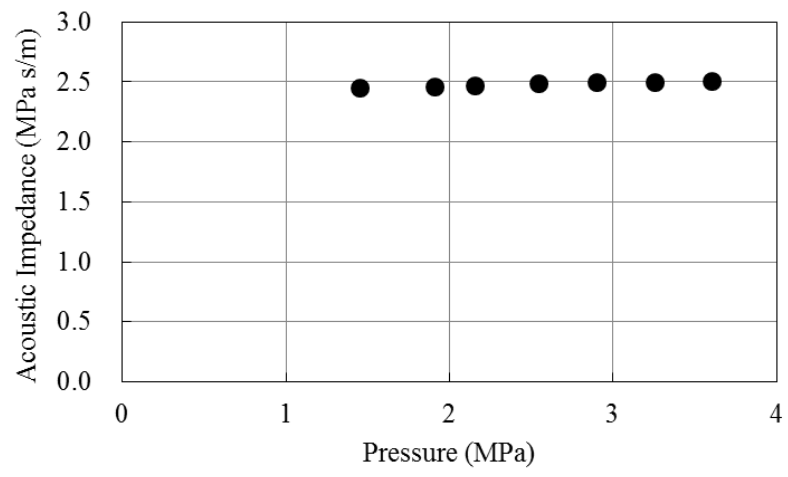

Fig. 8. Acoustic impedance of the coarse aluminum powder A32

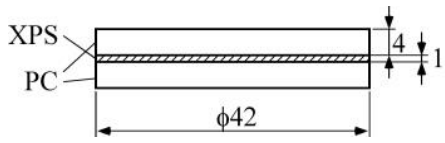

Fig. 9. Circular plate with a layer.

specimens is $2.3 \mathrm{MPa} \mathrm{s} / \mathrm{m}$ seen in Table 1 . This value matches that of the aluminum fine powder (A32) so this powder was used as the coupling. Two types of specimens were tested. One was a circular plate, $42 \mathrm{~mm}$ in diameter and $4.0 \mathrm{~mm}$ in thickness. The other was a circular plate with a layer of polystyrene form (XPS) as shown in Fig. 9.

Figure 9. shows the reflectogram of the polycarbonate plate. Echoes at $7 \mu$ s and $11 \mu \mathrm{s}$ are the reflections from the specimen. The echo height is lowest at $2.5 \mathrm{MPa}$ to $2.8 \mathrm{MPa}$ of pressure. This pressure equalizes the acoustic impedance of powder A32 and polycarbonate.

Figure 10. shows the reflectogram of the plate with a layer. The echo from the layer is much higher than the echoes from the specimen surfaces. Therefore, defects just below the surface of the test object can be detected by powder immersion testing. This characteristic enables defect detection of thin objects.

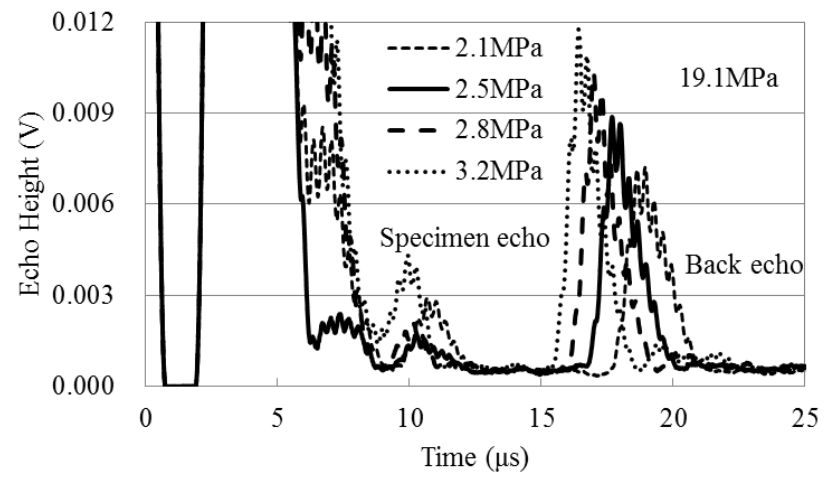

Fig. 10. Reflectogram of the polycarbonate plate.

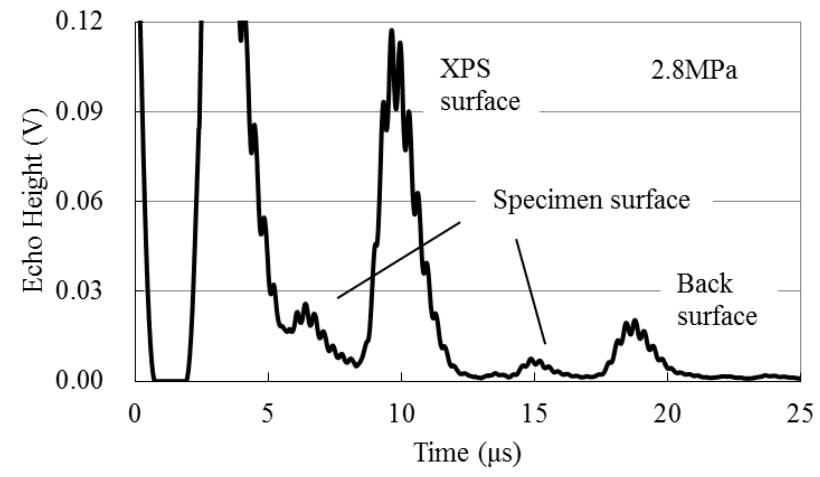

Fig. 11. Reflectogram of the plate with a layer.

\section{Conclusions}

Powder immersion testing has been developed and applied to a polycarbonate plate. According to the results we conclude as follows;

(1) The acoustic impedance of typical engineering plastics ranges from $2.1 \mathrm{MPa} \mathrm{s} / \mathrm{m}$ to $2.6 \mathrm{MPa} \mathrm{s} / \mathrm{m}$.

(2) Aluminum fine powder with a $1.0 \mu \mathrm{m}$ grain size has an acoustic impedance ranging from $1.8 \mathrm{MPa} \mathrm{s} / \mathrm{m}$ to 2.6 $\mathrm{MPa} \mathrm{s} / \mathrm{m}$.

(3) Powder immersion testing can be applied to typical engineering plastics.

(4) Powder immersion testing can detect defects just under the surface of the test objects. This characteristic enables defect detection of thin objects.

\section{References}

(1) Josef Krautkramer and Herbert Krautkramer : "Ultrasonic Testing of Materials," 4th Edition, Springer-Verlag, p. 187, 356, 1990

(2) W. T. Thomson, "Transmission of elastic waves through a stratified solid medium," Journal of Applied Physics, Vol. 21, No. 89, pp. 89-93, 1950 